\title{
Why Neurologists Should Remember About Antidepressants
}

\author{
Maxym Konyushok
}

\begin{abstract}
The importance of neurological diseases and mood disorders for human health and life, as well as for health care and society as a whole, cannot be overestimated. Nervous system pathologies such as stroke, neurodegenerative and demyelinating diseases, are one of the leading causes of death and disability in the world, and up to $60 \%$ of suicides are associated with depression. Antidepressants are used not only in psychiatric practice, but also in the practice of neurologists. Neurologists prescribe antidepressants to treat depression in neurological patients, chronic pain syndromes and neuropathic pain, panic attacks, eating disorders, premenstrual syndrome and for migraine prevention. The most difficult task for neurologists is to identify depressive disorders that often comorbid neurological diseases. The main property of antidepressants is the ability to increase depressed mood without increasing normal mood and without showing a stimulating effect. Antidepressants also have anti-anxiety, sedative, anti-phobic, anti-panic and somatic effects. When depression is detected in patients with neurological pathology, the prescription of antidepressants is justified not only from the psychiatric (relieving depression and reducing the risk of suicide), but also from the neurological point of view (reducing the risk of complications of neurological pathology and premature mortality from it).
\end{abstract}

Index Terms - depression, antidepressant, neurodegenerative disease, acute cerebrovascular disease, neuropathic pain, Parkinson's disease, bipolar depression, neurology, stoke, pain, attention deficit hyperactivity disorder, ADHD, disability.

\section{INTRODUCTION}

Neurological (especially cerebrovascular) diseases and depression have the greatest impact on human health and life. According to the WHO assessment based on the DisabilityAdjusted Life Year (DALY) criteria (reduction of working and socially fulfilling life expectancy), depression, cerebrovascular disease, and coronary heart disease are the most unfavorable [1]. In the nearest future, the burden of these diseases will be increased, with depression being the second leading cause of shortening a person's full life, and cerebrovascular disease the fourth [2].

Reducing the impact of risk factors and eliminating the manifestations of pathology of the nervous system and depression is the most important part in prevention mortality from them [2], [3]. The comorbidity of depression and neurological diseases (stroke, dementia, multiple sclerosis, Parkinson's disease and epilepsy) is a well-known fact in clinical practice, which has been repeatedly confirmed by studies [3]-[6]. According to current data, disorders such as depression and anxiety often occur in patients with the pathology of internal organs (from 20 to $70 \%$ of cases) [7] [9]. Depression is a common but usually unrecognized complication of cerebrovascular accident in 16-45\% patients with stroke [2], [10]. Depression is found in $30-40 \%$ of patients with dementia of both Alzheimer's and vascular type [11]. The comorbidity of depression and multiple sclerosis (MS) is over 50\% [12]. Depression is known to be as an important risk factor for stroke and its complications (including mortality), hypertension, arrhythmias and atherosclerosis [2], [13]. The risk of stroke is 1.43 times higher in people with depression and mortality from stroke in depression is 1.2 times higher at 12 and 24 months after an acute cerebrovascular disease.

Depression is a mental disorder characterized by low mood for two weeks, anhedonia (decreased interest or enjoyment of activities that are usually pleasant), lack of activity, energy and motivation, which leads to increased fatigue [9], [14], [15]. Other symptoms of depression include decreased confidence, pessimistic self-esteem, unreasonable selfcondemnation or excessive guilt, recurring thoughts of death or suicide, decreased ability to think, impaired concentration, agitation (motor restlessness, anxiety), any form of insomnia, decreased appetite and body weight. There may be depression with somatic disorders and dysfunctions that include heaviness in the heart, dry mouth, constipation, dry skin, tachycardia and fluctuations in blood pressure. More than half of patients with depression are not aware of low mood and express only somatic complaints, more often back pain, leg pain, etc., and the mechanism of pain (serotonin deficiency) is associated with the pathogenesis of depression) [14]-[16].

Depression may comorbid delirium [2], [17]. Delirium can develop at any age, but still more often in the elderly. At least $10 \%$ of elderly patients hospitalized with delirium, had delirium in previous admissions in 15 to $15 \%$ cases. Delirium also often occurs after surgery, in patients at home under the auspices of medical staff or among patients in the intensive care unit. If delirium develops in young people, it is usually the result of medication or a manifestation of some systemic, life-threatening condition. Delirium may be a common cause of psychotic symptoms such as hallucinations, bizarre delusions and thought-disorder, even in conditions such as schizophrenia, mania and depression, where delirium has traditionally been excluded by definition [16], [18], [19]. 
Neurological diseases may provoke onset and relapse of bipolar disorder [20]. Bipolar affective (mood) disorder used to be called manic-depressive psychosis. Bipolar affective (mood) disorder is a long-term and persistent inherited mental health disorder [21], [22]. This disorder is characterized by different mood swings. Depressive, manic and mixed states alternate, and sometimes there may be asymptomatic periods or periods when symptoms are mild. Often people with bipolar disorder do not seek help at the first symptoms. The reason for seeking help is usually a long and severe stage of depression. Periods of depression associated with bipolar disorder are more common than manic stages. Depressive stages in bipolar disorder occur the same way as depressive states in people with major depression. In the depressive stage, people with bipolar disorder experience severe fatigue, inability to concentrate, and sleep disorders are common.

Attention deficit hyperactivity disorder (ADHD) is a neurological and behavioral developmental disorder that begins in childhood [23]-[26]. ADHD is usually manifested by symptoms of difficulty concentrating, hyperactivity and poorly controlled impulsivity. Adults with ADHD may also have reduced intelligence and difficulty perceiving information. The diagnosis of ADHD in adults has a number of features. The boundaries of the diagnosis are so blurred that it can be unjustifiably imposed on many ordinary people who are dissatisfied with their ability to focus on boring and uninteresting work. The situation is exacerbated by the fact that the diagnosis is based on a person's subjective opinion about their ability to concentrate and perform tasks [27]. In addition, sometimes people who complain of ADHD symptoms may have bipolar disorder or depression. In this case, the prescription of conventional psychostimulants in the treatment of ADHD can only worsen the condition [27].

The other problem associated with ADHD is its treatment. Theoretically, all psychostimulants have the potential to cause sudden cardiac death and stroke [28]. It is not yet clear whether such a risk depends on which psychostimulant the patient is taking, whether he is exercising intensely, or on possible latent cardiac risk factors. Methylphenidate has been associated with sudden cardiac death in individuals with structural cardiac abnormalities, but there is no evidence that heart attacks are higher in those taking methylphenidate than in the general population [29]. This can lead to depression (post-stroke or post-infarction depression). General health, including dental health, functionality, daily activities and social adaptability will suffer [30], [31].

\section{DIAGNOSIS OF DEPRESSION}

To diagnose depression, the Beck Depression Inventory (BDI) is used, with which the patient can assess her/his own condition [32]. It requires 5-10 minutes, is very easy to fill, contains 21 items. A score of less than 10 points indicates remission, 14-19 indicates mild depression, 20-28 indicates moderate depression, and 29-63 indicates severe depression. A score of more than 24 points indicates the need for antidepressant treatment. Screening with the BDI should be performed in patients with cerebrovascular disorders, multiple sclerosis, post-stroke and traumatic brain injury and dementia of any type. According to current recommendations, antidepressants are the drugs of choice in the treatment of depressive disorders [7]-[9]. The main antidepressants' property is the ability to increase low mood without affecting normal mood and without showing a stimulating effect. Antidepressants also have anti-anxiety, sedative, anti-phobic and anti-panic effects and may act as painkillers.

Mild to moderate depression in neurological patients is successfully treated by neurologists. This has been made possible by the introduction into clinical practice of antidepressants from the group of selective serotonin reuptake inhibitors (SSRIs), such as sertraline that is characterized by minor adverse side effects on the nervous system and other internal organs [7]-[9].

Therefore, in the detection of depression in patients with neurological pathology, prescribing of antidepressants is justified not only from a psychiatric (relieving depression and reducing the risk of suicide), but also from a neurological point of view (reducing the risk of complications of neurological pathology and premature mortality). Studies have shown that about a third of outpatients require psychotropic drugs, especially antidepressants, due to anxiety and depression [1], [2], [7]-[10], [17].

\section{RELATIONSHIP BETWEEN DEPRESSION AND NEUROLOGICAL DISEASES}

Among neurological diseases a special place is occupied by cerebrovascular pathology. The most important risk factor for its development in depression is platelet activation. It has been established that patients with platelet depression show elevated levels of intracellular free calcium, hypersensitivity to serotonin (5-HT) and catecholamine receptors, hyperproduction of factor 4 and beta-thromboglobulin. This leads to increased vasoconstriction and platelet aggregation. The risk of platelet activation is further increased by high levels of catecholamines in the blood, which triggers the processes of aggregation and subsequent thrombosis, closely related to the development of acute cerebrovascular disease and ischemic stroke.

The association between nervous system pathology and depression is also explained by elevated levels of stress hormones (adrenaline and noradrenaline), manifested by sympathicotonia and glucocorticoids (cortisol), associated with dysfunction of the hypothalamic-pituitary-adrenal system. This can lead to heart rhythm problems, high blood pressure, an increased risk of blood clots, cerebral ischemia, increased blood cholesterol levels and a higher risk of developing atherosclerosis.

It is suggested that elevated level of serotonin in platelets is a factor contributing to thrombogenesis. This makes the use of SSRIs justified to reduce the risk of stroke in elderly patients with depression. ECG monitoring may reveal reduced heart rate variability, which contributes to the development of fatal ventricular arrhythmias. Arrhythmia in depression is a major risk factor for cerebrovascular microembolization, transient ischemic attacks and strokes.

Depression after a stroke significantly changes a person's behavior. It leads to decreased physical activity and dietary disorders. Patients do not follow the prescribed pharmacotherapy regimens and doctors' recommendations for lifestyle changes.

Diseases of the nervous system can lead to depressive 
disorders or exacerbate their course, increasing the risk of suicide. The relationship between the pathology of the neurological and internal diseases and mood disorders is multifactorial and complex. There are at least three types of causal relationships. The first type of depression is a direct consequence of brain damage (e.g., stroke and Parkinson's disease) [2], [5], [6]. The second type of depression is a psychological reaction to the disease or a manifestation of insufficient ability to overcome it. Finally, the third type of depression is a consequence of side effects of the treatment of the underlying disease (e.g., parkinsonism) [5], [6], [16]. In addition, autoimmune mechanisms and beta-interferon therapy may be important in multiple sclerosis [12].

One of the most dangerous problems of the modern medicine is the rapid increase in the incidence of acute cerebrovascular disease, as well as the high level of disability in affected patients. On the one hand, it has been proven that high levels of depression and anxiety are an independent risk factor for stroke, and on the other hand, depression is not only a frequent but also the least diagnosed complication of acute cerebrovascular disease. More than a third of patients after a stroke suffer from depression. It involves brain damage that causes serotonin dysfunction and distress related to loss of motor skills and speech. In addition, depression is more often associated with the localization of stroke in the left anterior part of the brain and basal ganglia, as well as in the limbic system, which regulates emotions.

Depression is diagnosed by neurologists and general practice physicians in approximately $40 \%$ of patients who have had a stroke, but only $10-15 \%$ of them receive antidepressants [2]. Lack of timely diagnosis and treatment of post-stroke depression reduces the quality of life of patients, negatively affects their motor rehabilitation, complicates the correction of hypertension, leads to permanent disability and suicide attempts. It is important to treat depression as an additional and independent risk factor. It has been shown that depression in the elderly can be both a risk factor and the first manifestation of dementia. Severe depression requires not just a short course of treatment, but maintenance therapy to prevent recurrence of depression and complications of the underlying disorder. In the process of adequate therapy, complete reversibility of clinical manifestation of depression is possible. In patients after stroke, even without clinical manifestations of depression, the commencement of antidepressants improves prognosis and survival, which may be associated with physical (hidden) nature of depression and the impact of drugs on specific links in the pathogenesis of cerebrovascular pathology.

\section{TREATMENT OF DEPRESSION IN NEUROLOGICAL PATIENTS}

The first drugs to be used in depressed patients with neurological diseases were from the class of Tricyclic antidepressants (TCAs). In addition, atypical antidepressants such as trazodone were used. But, unfortunately, these drugs were characterized by a high frequency of side effects, even such serious as delirium, due to cholinergic properties [7], [8], [33], [34]. A few meta-analyses proved the effectiveness of antidepressants in the treatment of post-stroke depression [2], [35]. Long term therapy is more effective: the benefits of treatment appear on the $3^{\text {rd }}$ and $4^{\text {th }}$ week and intensify on the $8^{\text {th }}$ week. SSRIs, in particular sertraline, are the drugs of choice in neurology.

SSRI class of antidepressants is a group of choice for the treatment of depression in the elderly [7], [8]. They are characterized by rapid development of therapeutic effect, well tolerance, easy in dosing and do not affect antianginal, antiarrhythmic, antihypertensive and antithrombotic therapy. SSRIs have both antidepressant and antianxiety effects, so they are effective in patients with depression comorbid anxiety symptoms (panic attacks and phobias). These agents have proven effectiveness in treating depression in elderly patients, Alzheimer's and Parkinson's disease, pathological crying and laughing in evidence-based research [2], [5], [6], [35].

\section{TREATMENT OF DEPRESSION IN PATIENTS WITH DEMENTIA}

Depression is often found in patients with various types of dementia: due to Alzheimer's disease (AD), after multiple strokes or frontotemporal dementia. All SSRIs have been shown to be effective in combining depression and dementia, but vascular dementia is known to be less amenable to treatment, and fluoxetine sometimes worsens the condition of such patients [2], [9], [11], [36].

The use of SSRIs in AD, in particular, sertraline is more effective at a dosage of $150 \mathrm{mg}$ for 3 months [37], [38]. The positive effect of sertraline on motor and cognitive functions of patients was also noted. In addition, the possibility of the effect of serotonergic antidepressants on neurogenesis and slowing of Alzheimer's dementia has been discussed in recent years.

Vascular dementia causes depression with greater frequency and severity [38]. This allowed the introduction of a special term "vascular depression", which develops on the background of hypertension and is characterized by apathy, inhibition, c'ognitive impairment, MRI signs of subcortical lesions of the gray and white matter of the brain. For the treatment of "vascular depression" it is recommended to use those antidepressants that do not interact with antihypertensive drugs.

\section{TREATMENT OF DEPRESSION IN PATIENTS WITH PARKINSON'S DISEASE}

Parkinson's disease (PD) develops more often in the elderly (55-66 years) and is characterized by motor and psychiatric symptoms [5], [6]. This is the second most common neurodegenerative disorder after AD. The purpose of pharmacotherapy is usually motor disorders, while psychiatric symptoms (dementia, psychosis, depression) are considered the most disabling and unrecognizable manifestations of the disease [5], [6], [39]. The widespread use of dopaminergic agents for the treatment of Parkinson's disease has revealed the presence of pathological gambling disorder as an uncommon, but serious, adverse reaction to these agents [40], [41]. Symptoms of depression occur in more than half of patients with PD. More than $40 \%$ of depressed patients with PD do not receive specific treatment 
[5], [6]. At the same time, depression increases the likelihood of prescribing antiparkinsonian drugs, as many complaints caused by depression (fatigue, motor retardation) are perceived as symptoms of PD. Pramipexole may be affective for antidepressant treatment [5], [6].

The development of depression is associated with neurodegeneration of the brainstem, which damages the central serotonergic system. That is why serotonergic antidepressants (including SSRIs) are able to most effectively eliminate the manifestations of depression in PD. Prescribing of SSRIs may significantly improve mood in $70 \%$ of subjects, reduced akinesia, which is associated with the ability of SSRIs to block dopamine reuptake. In this case, for example, sertraline, in contrast to fluoxetine and paroxetine, did not cause an increase in extrapyramidal symptoms.

\section{TREATMENT OF DEPRESSION IN PATIENTS WITH MULTIPLE SCLEROSIS (MS)}

Multiple sclerosis (MS) is the most common demyelinating disease [12]. More than $50 \%$ of MS patients also suffer from depression. The use of antidepressants in patients with MS comorbid depression is highly recommended [12], [42]. SSRIs can alleviate the symptoms of depression in MS, but also affect the state of the immune system. In addition, serotonergic antidepressant therapy induces non-specific and antigen-specific suppression of Tlymphocytes, which can be used as an additional strategy in the treatment of MS.

\section{TREATMENT OF DEPRESSION IN PATIENTS WITH EPILEPSY}

Epilepsy is considered one of the most common chronic neurological diseases in humans [43], [44]. Epilepsy is a chronic disorder of the brain that is characterized by recurrent seizures. Depression occurs in more than $25 \%$ of patients with seizures, but $60 \%$ of such patients are not diagnosed within a year or more [44]. The administration of SSRIs to depressed patients with partial epilepsy has led to an improvement in their condition and quality of life without affecting the frequency of seizures and interactions with anticonvulsants. On the other hand, many antidepressants of SSRI class (fluoxetine, paroxetine) affect the metabolism of antiepileptic drugs, but serotonin-norepinephrine reuptake inhibitors (SNRIs), e.g., venlafaxine, in high doses can cause seizures in $14 \%$ of patients [8], [9].

\section{BACK PAIN: OSTEOCHONDROSIS AND SOMATIZATION DEPRESSION}

Back pain is one of the most common reasons for seeing a neurologist. Most often in such cases it is not possible to identify any specific cause of pain, and the patient is diagnosed with osteochondrosis. In many cases, such complaints are actually a manifestation of somatization (masked) depression, which occurs in more than a third of neurological patients. Additional investigations for the causes of pain (e.g., CT or MRI) are ineffective, and patients are sometimes even ready for surgery or second opinion referral.
The use of antidepressants in dorsalgia (spine pain) is pathogenetically justified as due to its effect on serotonergic transmission they can affect the nociception (pain system of the brain).

\section{TREATMENT OF DEPRESSION IN PATIENTS WITH AUTISM SPECTRUM DISORDER}

Autism is not a disease, but it is a condition with which a person is born. It cannot be cured, but over time we can adjust and adapt a person to social life [45]. Autism manifests itself in developmental delay and unwillingness to contact the outside world. It cannot be diagnosed immediately at birth. It manifests itself later at the physiological level. We can recognize it by observing the behavior and reactions of the baby. However, due to misdiagnosis, children with autism are diagnosed with schizophrenia. The main task of pharmacology therapy in autism is to eliminate certain undesirable symptoms that reduce the effectiveness of educational and psychological correction, such as aggression, stereotypes, negativism, and motor disinhibition. In addition, some drugs stimulate the integrative activity of the brain, activate the processes of memory and thinking.

It is recommended to prescribe antidepressants with sedative properties to preschool children with a diagnosis of autism, in particular, tricyclic antidepressant - amitriptyline [46]. It is believed that amitriptyline may help improve interaction with autistic patient. Amitriptyline may also increase patient's interest in the surrounding world. Antidepressants cannot cure autism, but can improve patient's behaviour during psychological and behavioural treatment. The disadvantage of amitriptyline is a large number of side effects, such as, drowsiness, decreased blood pressure, dry mouth, nausea, constipation and urinary retention. The drug should only be prescribed under EEG control as it may provoke the onset of convulsions.

\section{CONCLUSIONS}

Depression significantly affects the health and lives of patients with neurological pathology. Knowledge of risk factors, mechanisms of development and clinical presentation of this condition in combination with appropriate use of modern antidepressants allows practicing neurologists not only to reduce duration of depression and to alleviate patient sufferings, but also to prevent suicide, to improve the prognosis of the basic disease and to reduce lethality. An adequate use of antidepressants may improve the quality of life and maintain full social functioning of the affected patient.

\section{REFERENCES}

[1] Baert, I., Feys, H., Daly, D., Troosters, T., \& Vanlandewijck, Y. (2012). Are patients 1 year post-stroke active enough to improve their physical health?. Disability and rehabilitation, 34(7), 574-580.

[2] Tsarkov, A., \& Petlovanyi, P. (2019). Neuropsychiatric Aspects of a Common Problem: Stroke. European Journal of Medical and Health Sciences, 1(3)

[3] Tsarkov, A., \& Petlovanyi, P. The Role of Lamotrigine in the Treatment of Bipolar Depression.

[4] Shibata, M., \& Suzuki, N. (2017). Exploring the role of microglia in cortical spreading depression in neurological disease. Journal of Cerebral Blood Flow \& Metabolism, 37(4), 1182-1191. 
[5] Anatolii, T., \& Petro, P. (2020). Use of pramipexole in neuropsychiatry. World Journal of Advanced Research and Reviews, 7(2), 82-88.

[6] Tsarkov, A., \& Petlovanyi, P. (2020). Use of pramipexole in neuropsychiatry. World Journal of Advanced Research and Reviews, $7(2), 82-88$

[7] Lebedyn, Z. (2019). The Use of Antidepressants by General Practitioners and Psychiatrists (Personal Experience).

[8] Lebedyn, Z. (2020). Antidepressants In General Practice And Psychiatry. European Journal of Medical and Health Sciences, 2(3). https://doi.org/10.24018/ejmed.2020.2.3.318.

[9] Lebedyn, Z. (2020). Recommendations For The Management Of Patients With Depression In General Clinical Practice. European Journal of Medical and Health Sciences, 2(4). https://doi.org/10.24018/ejmed.2020.2.4.416.

[10] Aström, M., Adolfsson, R., \& Asplund, K. (1993). Major depression in stroke patients. A 3-year longitudinal study. Stroke, 24(7), 976-982.

[11] Korczyn, A. D., \& Halperin, I. (2009). Depression and dementia. Journal of the neurological sciences, 283(1-2), 139-142.

[12] Siegert, R. J., \& Abernethy, D. A. (2005). Depression in multiple sclerosis: a review. Journal of Neurology, Neurosurgery \& Psychiatry, 76(4), 469-475.

[13] Thomas, A. J., Kalaria, R. N., \& T O’Brien, J. (2004). Depression and vascular disease: what is the relationship?. Journal of affective disorders, 79(1-3), 81-95.

[14] Petlovanyi, P., \& Tsarkov, A. (2017). Depressive Disorder in Child Psychiatric Practice: A Case Report. Health Press Zambia Bull, 1(5), 9.

[15] Tsarkov, A., \& Petlovanyi, P. Depressive Disorder in Child Psychiatric Practice: A. The Health Press, 9.

[16] Petlovanyi, P., \& Tsarkov, A. Child Schizophrenia: Theory and Practice.

[17] Rincon, H. G., Granados, M., Unutzer, J., Gomez, M., Duran, R., Badiel, M., ... \& Florez, N. (2001). Prevalence, detection and treatmen of anxiety, depression, and delirium in the adult critical care unit. Psychosomatics, 42(5), 391-396.

[18] Anatolii, T., Patrick, M., \& Petro, P. (2020). Uncommon presentation: Folie à deux (Case study). World Journal of Advanced Research and Reviews, 6(3), 043-049.

[19] Tsarkov, A., Msoni, P., \& Petlovanyi, P. (2020). Uncommon presentation: Folie à deux (Case study). World Journal of Advanced Research and Reviews, 6(3), 043-049.

[20] Schneck, C. D. (2002). Bipolar disorder in neurologic illness. Current Treatment Options in Neurology, 4(6), 477-486.

[21] Tsarkov, A., \& Petlovanyi, P. (2016). Bipolar disorder in child psychiatric practice.

[22] Tsarkov, A., \& Petlovanyi, P. (2016). Bipolar Disorder in Child Psychiatric Practice: A Case Report. Medical Journal of Zambia, 43(1), 41-46.

[23] Sonuga-Barke, E. J., Brandeis, D., Cortese, S., Daley, D., Ferrin, M. Holtmann, M., ... \& Dittmann, R. W. (2013). Nonpharmacological interventions for ADHD: systematic review and meta-analyses of randomized controlled trials of dietary and psychological treatments. American Journal of Psychiatry, 170(3), 275-289.

[24] Petro, P., \& Anatolii, T. (2020). Practical guide and some recommendations for the diagnosis and management of Attention deficit hyperactivity disorder (ADHD). World Journal of Advanced Research and Reviews, 6(3), 257-261.

[25] Tsarkov, A., \& Petlovanyi, P. (2017). Omega-3 Fatty Acids as an Alternative Treatment for Children with Attention Deficit Hyperactivity Disorder. Imperial Journal of Interdisciplinary Research (IJIR), 3, 1378-1380.

[26] Petlovanyi, P., \& Tsarkov, A. (2020). Practical guide and some recommendations for the diagnosis and management of Attention deficit hyperactivity disorder (ADHD). World Journal of Advanced Research and Reviews, 6(3), 257-261.

[27] Batstra, L., \& Frances, A. (2012). DSM-5 further inflates attention deficit hyperactivity disorder. The Journal of nervous and mental disease, 200(6), 486-488.

[28] Groenman, A. P., Schweren, L. J., Dietrich, A., \& Hoekstra, P. J (2017). An update on the safety of psychostimulants for the treatment of attention-deficit/hyperactivity disorder. Expert Opinion on Drug Safety, 16(4), 455-464

[29] Bange, F., Le Heuzey, M. F., Acquaviva, E., Delorme, R., \& Mouren, M. C. (2013). Cardiovascular risks and management during Attention Deficit Hyperactivity Disorder treatment with methylphenidate. Archives de pediatrie: organe officiel de la Societe francaise de pediatrie, 21(1), 108-112.

[30] Ladwig, K. H., Roll, G., Breithard, G., Budde, T., \& Borggrefe, M. (1994). Post-infarction depression and incomplete recovery 6 months after acute myocardial infarction. The Lancet, 343(8888), 20-23.
[31] Phiri, C., Tsarkov, A., Petlovanyi, P., \& Lingenda, G. Factor Contributing To Oral Diseases and Treatment Needs amongst Mental Patients at Chainama Hills College Hospital, Lusaka, Zambia.

[32] Wedding, U., Koch, A., Röhrig, B., Pientka, L., Sauer, H., Höffken, K. \& Maurer, I. (2007). Requestioning depression in patients with cancer: contribution of somatic and affective symptoms to Beck's Depression Inventory. Annals of Oncology, 18(11), 1875-1881.

[33] Thanacoody, H. R., \& Thomas, S. H. (2005). Tricyclic antidepressant poisoning. Toxicological reviews, 24(3), 205-214.

[34] Raffaele, R., Rampello, L., Vecchio, I., Tornali, C., \& Malaguarnera M. (1996). Trazodone therapy of the post-stroke depression. Archives of gerontology and geriatrics, 22, 217-220.

[35] Paolucci, S. (2017). Advances in antidepressants for treating poststroke depression. Expert opinion on pharmacotherapy, 18(10), 1011 1017.

[36] Seitz, D. P., Adunuri, N., Gill, S. S., Gruneir, A., Herrmann, N., \& Rochon, P. (2011). Antidepressants for agitation and psychosis in dementia. Cochrane Database of Systematic Reviews, (2).

[37] Magai, C., Kennedy, G., Cohen, C. I., \& Gomberg, D. (2000). A controlled clinical trial of sertraline in the treatment of depression in nursing home patients with late-stage Alzheimer's disease. The American Journal of Geriatric Psychiatry, 8(1), 66-74.

[38] Diniz, B. S., Butters, M. A., Albert, S. M., Dew, M. A., \& Reynolds, C. F. (2013). Late-life depression and risk of vascular dementia and Alzheimer's disease: systematic review and meta-analysis of community-based cohort studies. The British Journal of Psychiatry, 202(5), 329-335

[39] Schrag, A., Hovris, A., Morley, D., Quinn, N., \& Jahanshahi, M. (2006). Caregiver-burden in Parkinson's disease is closely associated with psychiatric symptoms, falls, and disability. Parkinsonism \& related disorders, $12(1), 35-41$.

[40] Tsarkov, A., \& Petlovanyi, P. (2017). Pathological Gambling: The Old Problem of the Modern World. Imperial Journal of Interdisciplinary Research, 3(8), 216-221.

[41] Lanteri, P. F., Leguia, A., Doladé, N. G., García, G. C., \& Figueras, A. (2018). Drug-induced gambling disorder: A not so rare but underreported condition. Psychiatry research, 269, 593-595.

[42] Cetin, K., Johnson, K. L., Ehde, D. M., Kuehn, C. M., Amtmann, D. \& Kraft, G. H. (2007). Antidepressant use in multiple sclerosis: epidemiologic study of a large community sample. Multiple Sclerosis Journal, 13(8), 1046-1053.

[43] Lebedyn, Z. (2020). Diagnostic Procedure and Therapeutic Approaches in Nonconvulsive Status Epilepticus (NCSE). European Journal of Medical and Health Sciences, 2(4). https://doi.org/10.24018/ejmed.2020.2.4.426

[44] Maguire, M. J., Weston, J., Singh, J., \& Marson, A. G. (2014) Antidepressants for people with epilepsy and depression. Cochrane Database of Systematic Reviews, (12).

[45] Konyushok, M. (2020). Autism Spectrum Disorder in Adult Patients: Clinical Presentation And Management. European Journal of Medical and Health Sciences, 2(4) https://doi.org/10.24018/ejmed.2020.2.4.428

[46] Stewart, M. E., Barnard, L., Pearson, J., Hasan, R., \& O’Brien, G. (2006). Presentation of depression in autism and Asperger syndrome: A review. Autism, 10(1), 103-116 